\title{
Investigation of the Role of Glypican 3 in Liver Regeneration and Hepatocyte Proliferation
}

Bowen Liu, Shirish Paranjpe, William C. Bowen, Aaron W. Bell, Jian-Hua Luo, Yan-Ping Yu, Wendy M. Mars, and George K. Michalopoulos

From the Division of Experimental Patbology, Department of Pathology, University of Pittsburgh School of Medicine,

Pittsburgh, Pennsylvania

Glypicans are heparan sulfate proteoglycans that are bound to the cell surface by glycosylphosphatidylinositol. While six members of the glypican family are known in mammals, our study focused on glypican 3 (GPC3). Loss-of-function mutations of GPC3 result in the Simpson-Golabi-Behmel syndrome, an X-linked disorder characterized by pre- and postnatal liver and other organ overgrowth. GPC3 is overexpressed in human hepatocellular carcinoma; however, its role in normal liver regeneration and hepatocyte proliferation is unknown. Here we investigated the role of GPC3 in hepatocyte proliferation. GPC3 mRNA and protein levels begin to increase 2 days after hepatectomy with peak expression levels by day 5 . In hepatocyte cultures, GPC3 reaches a plateau when hepatocyte proliferation decreases. In vitro studies using Morpholino oligonucleotides showed that blocking GPC3 expression promoted hepatocyte growth. Yeast two-hybrid assays revealed that GPC3 interacts with CD81, a member of the tetraspanin family that is reported to be involved in hepatitis $\mathrm{C}$ virus infection and cell proliferation. We found that CD81 levels also increased 2 days after partial hepatectomy and toward the end of regeneration. Immunofluorescence showed that CD81 and GPC3 colocalize by 2 and 6 days after hepatectomy. Co-immunoprecipitation validated the interaction of GPC3 and CD81. Our results indicate that GPC3 may be a negative regulator of liver regeneration and hepatocyte proliferation, and that this regulation may involve CD81. (Am J Pathol 2009, 175:717-724; DOI: 10.2353/ajpath.2009.081129)

Glypican 3 (GPC3) belongs to a family of glycosylphosphatidylinositol-anchored, cell-surface heparan sulfate proteoglycans. ${ }^{1}$ Six glypicans have been identified in mammals so far (GPC1 to GPC6) and two members of this family have been found in Drosophila (dally and dlp). ${ }^{2,3}$ Although the homology of amino acids between glypican members is moderate, all glypicans are approximately 60 to $70 \mathrm{kd}$ in size and share a characteristic pattern of 14 conserved cysteine residues. ${ }^{4}$ Intact glypicans are decorated with heparan sulfate (HS), which is located in the last 50 amino acids of the $\mathrm{C}$ terminus, placing the HS chains close to the cell membrane. ${ }^{5}$

GPC3 is located on the X chromosome, and is highly expressed during embryogenesis and organogenesis., ${ }^{2,6}$ In the adult, on the other hand, GPC3 can only be detected in a limited number of tissues, including the lung, ovaries, mammary epithelium, and mesothelium. ${ }^{2,7}$ GPC3 is highly up-regulated in hepatocellular carcinoma, one of the most common solid malignancies in the world that accounts for about 1 million deaths each year. ${ }^{8}$ Our previous study and others have shown that GPC3 is highly up-regulated in hepatocellular carcinoma and hepatoblastoma, but not in normal liver or tissue adjacent to tumors. ${ }^{8,9}$ The soluble form of GPC3 was also identified in the serum of patients with hepatocellular carcinomas, and can be used as a serological test for the diagnosis of hepatocellular carcinoma. ${ }^{10,11}$ GPC3 is also involved in cell proliferation in some hepatoma cell lines. ${ }^{12}$

The stage- and tissue-specific pattern of expression suggests that GPC3 is involved in morphogenesis and development. It is reported that a loss-of -function mutation in the GPC3 gene causes Simpson-Golabi-Behmel syndrome, an $\mathrm{X}$-linked disorder characterized by preand postnatal overgrowth, increased risk of embryonic tumors during early childhood, and numerous visceral and skeletal anomalies. ${ }^{13,14}$ The involvement of GPC3 in Simpson-Golabi-Behmel syndrome was confirmed by the generation of GPC3-deficient mice (GPC3-/-), since these mice display some of the phenotypic features of

Supported by National Institutes of Health grants CA30241 and CA35373 (to G.K.M.) and the Rangos Fund for Enhancement of Pathology Research.

Accepted for publication April 21, 2009.

Address reprint requests to George K. Michalopoulos, Department of Pathology, University of Pittsburgh School of Medicine, S-410 Biomedical Science Tower, Pittsburgh, PA 15261. E-mail: michalopoulosgk@ upmc.edu. 
Simpson-Golabi-Behmel syndrome, including developmental overgrowth ( 30\%), general enlargement of multiple organ including liver, respiratory infections, cystic kidneys, etc. ${ }^{15}$ These findings suggest that GPC3 plays a negative growth regulatory role despite its over-expression in liver cancer.

Since loss of function in GPC3 in Simpson-GolabiBehmel syndrome leads to overgrowth of many organs including liver in humans and mice, it is reasonable to speculate that GPC3 functions as a growth inhibitor in the liver. Given the overexpression of GPC3 in human liver cancer, we wanted to study the role of GPC3 in hepatocyte growth regulation. To that end, we used the rodent model of liver regeneration after partial hepatectomy $(\mathrm{PHx})$ in which hepatocyte growth dynamics are well characterized. ${ }^{16,17}$ We also used hepatocyte primary cultures, in which we and others have characterized the hepatocyte growth cycle under the influence of hepatocyte growth factor (HGF) and epidermal growth factor (EGF) ${ }^{18}$ In liver regeneration after $2 / 3 \mathrm{PHx},{ }^{19}$ specific rat liver lobes are removed intact (about 2/3 of the total mass) without damage to the lobes left behind. The residual lobes enlarge to make up for the mass of the removed lobes, though the resected lobes never grow back. ${ }^{19}$ Following liver $\mathrm{PHx}$, hepatocytes enter into the cell cycle from their habitual quiescent phase and proliferate to restore normal hepatic mass and hepatic functional capacity. The whole process lasts 5 to 7 days in rats, during which the hepatocytes divide first at about 24 hours post-PHx, followed by the biliary ductular cells, then the Kupffer cells and stellate cells, and finally the endothelial cells. ${ }^{17}$ Details of this process and the dynamics of proliferation of different cell types, role of growth factors and cytokines have been described. ${ }^{17}$

Our results show that GPC3 protein and RNA levels are increased in the later stages of liver regeneration, as well as in the later stages of growth cessation in HGF and EGF stimulated hepatocyte cultures. When GPC3 protein is knocked down using Morpholino oligonucleotides (oligos), hepatocyte growth is promoted at the end of proliferation. Thus we hypothesize that GPC3 could negatively regulate liver regeneration and hepatocyte proliferation.

To investigate the potential proteins that could interact with GPC3, a yeast two-hybrid assay was performed, and several interesting proteins, including CD81, were found to interact with GPC3. CD81, also called TAPA-1 (target of an antiproliferative antibody) is a widely expressed cell surface tetraspanin involved in an astonishing variety of biological responses. ${ }^{20}$ It has been cloned independently several times for different functional effects and is reported to influence adhesion, morphology, activation, proliferation, and differentiation of $\mathrm{B}, \mathrm{T}$, and other cells. ${ }^{21,22}$ CD81 is also reported to interact with HCV glycoprotein E2 and assist with HCV infection in hepatocytes. ${ }^{23}$ In our research, CD81 RNA and protein levels changed in the same manner as GPC3. In addition, co-immunofluorescence and co-immunoprecipitation were performed that verified the interaction and localization of GPC3 and CD81 in rat liver, suggesting that CD81 interaction is likely to form an important relationship with ligands to GPC3 function.

\section{Materials and Methods}

\section{Animals and Partial Hepatectomy Model}

Male Fisher344 rats (150-200 g) were purchased from Charles River Laboratories (Frederick, MD). Animals were allowed access to food and water ad libitum. Isoflurane inhalation (Baxter, IL) was used to anesthetize animals. In the $\mathrm{PHx}$ model, the median and left lateral lobes (accounting for $2 / 3$ of the total liver mass) were resected. ${ }^{24}$ The remaining liver lobes were obtained from defined time points after $\mathrm{PHx}$ as 1, 2, 3, 4, 5, 6, and 7 days. All liver samples were promptly frozen in liquid nitrogen and stored at $-80^{\circ} \mathrm{C}$. Fresh rat liver tissue was placed in Tissue-Tek OCT embedding compound and frozen on dry ice and stored at $-80^{\circ} \mathrm{C}$. All animals were housed in the animal facility of the University of Pittsburgh, and all procedures performed on these rats were approved under the IACUC protocol 0805741-1 and conducted according to National Institutes of Health guidelines.

\section{Cell Culture and Proliferation Assay}

Hepatocytes were isolated from normal Fisher 344 rats by an adaptation of Seglen's calcium two-step collagenase perfusion technique ${ }^{25}$ as previously described from our laboratory. ${ }^{26}$ Isolated rat hepatocytes were added to collagen-coated six-well plates (BD Biosciences, CA). Each well contained 200,000 freshly isolated hepatocytes in $1 \mathrm{ml}$ of hepatocyte growth medium supplemented with HGF (40 $\mathrm{ng} / \mathrm{ml})$ and EGF $(20 \mathrm{ng} / \mathrm{ml})$. $\left.{ }^{3} \mathrm{H}\right]$ thymidine was added to the medium for 48 hours at a concentration of $2.5 \mu \mathrm{Ci} / \mathrm{ml}$. The medium was removed at defined time points, and hepatocytes were fixed with ice-cold $5 \%$ trichloroacetic acid. Then trichloroacetic acid was removed and the plates were washed in running tap water and air dried completely. Five hundred microliters of $0.33 \mathrm{~N} \mathrm{NaOH}$ was added to each well for 30 minutes to solubilize the cells. The solution was transferred into a new tube, and $166 \mu$ of $20 \%$ trichloroacetic acid/1.2 N HCl was added for precipitation. The tubes were centrifuged at maximum speed for 10 minutes, and the pellets were re-dissolved in $500 \mu$ of $0.33 \mathrm{~N} \mathrm{NaOH}$. A 200- $\mu$ l aliquot was used to measure cpm/dpm in a Beckman LS6000IC scintillation counter (Beckman Coulter, CA), and $100 \mu \mathrm{l}$ was used to determine the optical density value of total DNA.

\section{RNA Extraction and Semiquantitative Reverse}

\section{Transcription-Polymerase Chain Reaction (RT-PCR)}

RNA was extracted from frozen liver tissues with Trizol (Invitrogen, CA) according to the manufacturer's instructions. RNA, $5 \mu \mathrm{g}$, was reverse-transcribed to complementary DNA (cDNA) with SuperScript III reverse transcriptase (Invitrogen, CA) according to the standard protocol. Standard PCR was performed with Taq polymerase (Qiagen, CA). The primers used to perform PCR are: GPC3-forward: 5'GGTGACGGCATGATGAAAGTGAAG-3'; GPC3-reverse: 5'-TGGTGATCTCGTTGTCCTTCTGAT-3'; CD81-forward: 
5'-CGCGGTACCATGGGGGTGGAGGGCTGCAC-3'; CD81reverse: 5'-CCGGAATTCTCAGTACACGGAGCTGTTCCGG-3'. PCR products were resolved on $2 \%$ agarose gels and visualized with ethidium bromide staining, and digital images were quantitated using National Institutes of Health ImageJ software.

\section{Protein Extraction and Western Blotting}

Frozen liver tissue and hepatocytes were homogenized in RIPA buffer $(50 \mathrm{mmol} / \mathrm{L}$ Tris-HCl, pH 7.4, $150 \mathrm{mmol} / \mathrm{L}$ $\mathrm{NaCl}, 0.1 \%$ sodium dodecyl sulfate) supplemented with proteinase inhibitors. For HS chain elimination, $100 \mu \mathrm{g}$ of protein extract from freshly isolated rat hepatocytes and total rat liver was treated with 5 microunits of heparitinase enzyme (Seikagaku, Tokyo, Japan) and $1 \mathrm{mmol} / \mathrm{L} \mathrm{CaCl}_{2}$ for 3 hours. ${ }^{27}$ Protein samples $(20 \mu \mathrm{g})$ were resolved on 4 to 12\% NuPAGE Bis-Tris gels with 1X 3-(N-morpholino) propanesulfonic acid running buffer (Invitrogen, CA) and then transferred to Immobilon-P membranes (Millipore, MA) in NuPAGE transfer buffer containing 10\% methanol. Membranes were stained with $0.2 \%$ Ponceau Red to verify loading and transfer efficiency. Membranes were then probed with primary and secondary antibodies in TBST buffer (Tris-buffered saline-Tween 20) containing $5 \%$ nonfat milk, then processed with SuperSignal West Pico chemiluminescence substrate (Pierce, IL) and exposed to a X-ray film (Lab Product Sales, NY).

\section{Knocking Down GPC3 using Morpholino Oligos}

Morpholino antisense oligos targeted to translational start sites of rat GPC3 were designed and synthesized by Gene Tools, LLC (Philomath, OR). The Morpholino oligo sequence complementary to the translational blocking target GPC3 is: 5'-CACGGTCCCGGCCATCCTGCTTCTT-3'. The Morpholino was complexed with the Endo-Porter delivery reagent, which is used to deliver "bare" oligos by an endocytosismediated process. ${ }^{28}$ Rat hepatocytes were isolated and plated into collagen-coated six-well plates. In brief, $1 \mathrm{ml}$ of hepatocyte growth medium with HGF and EGF was combined with $20 \mu \mathrm{l}$ of $1 \mathrm{mmol} / \mathrm{L}$ Morpholino oligos and $5 \mu \mathrm{l}$ of $1 \mathrm{mmol} / \mathrm{L}$ Endo-Porter dissolved in dimethyl sulfoxide and applied to cell cultures in six-well plates for 48 hours. EndoPorter solution alone was added as a negative control. After the allotted time period, the medium was removed and replaced with fresh hepatocyte growth medium with HGF and EGF. For a 6-day experiment, Morpholino oligos and Endo-Porter reagent were added at days 2 and day 4 . $\left[{ }^{3} \mathrm{H}\right]$ thymidine assay was performed as described above. All transfection experiments were performed in triplicate. GPC3 protein level for each time point was determined by Western blotting.

\section{Yeast Two-Hybrid Assay}

The Matchmaker GAL4 yeast two-hybrid system (Clontech, CA) was used for identification of GPC3-binding protein according to standard protocol. ${ }^{29}$ As bait for screening, the vector pGBKT7 expressing a fusion pro- tein composed of full-length rat GPC3 cDNA linked to the DNA-binding domain was constructed. A rat liver CDNA library was cloned into pACT2 vector containing a GAL4 activation domain. Both of the vectors were used to transform the AH109 yeast strain. Positive clones were selected on SD/-Ade/-His/-Leu/-Trp high stringency YPDA plates with X-gal. Plasmids from positive clones were subsequently isolated from the yeast, transferred to Escherichia coli Top10 competent cells (Invitrogen, CA), and sequenced. The GenBank/NCBI databases were screened for similar sequences using BLAST search.

\section{Co-Immunoprecipitation}

Normal rat liver tissue was lysed in RIPA buffer as described above. For immunoprecipitation studies, $500 \mu \mathrm{g}$ of protein lysates were diluted to a final volume of $500 \mu \mathrm{l}$ and precleared by incubating with $1 \mu \mathrm{g}$ of hamster IgG (Santa Cruz, CA) and $20 \mu \mathrm{l}$ of agarose A/G plus beads (Santa Cruz, CA) for 1 hour at room temperature. Protein complexes were immunoprecipitated from cleared lysates with $1 \mu \mathrm{g}$ of anti-CD81 monoclonal antibody (GeneTex, TX) overnight at $4^{\circ} \mathrm{C}$, followed by a 5 -hour incubation at $4^{\circ} \mathrm{C}$ with agarose $A / G$ plus beads. Immune complexes were then collected and washed three times with RIPA buffer before resuspension in $4 \mathrm{X}$ sodium dodecyl sulfate-polyacrylamide gel electrophoresis sample buffer and $1 \mathrm{X}$ reducing reagent (Invitrogen, CA). Proteins from immunoprecipitated samples or $20 \mu \mathrm{g}$ of crude liver protein were separated by sodium dodecyl sulfate-polyacrylamide gel electrophoresis and subjected to Western blotting using antibody against GPC3 (Aviva, CA). All protein bands were detected using Western blotting as described above.

\section{Co-Immunofluorescence}

Frozen rat liver sections (5 mm) were fixed in ice-cold 100\% acetone for 5 minutes, blocked with $5 \%$ normal goat serum in phosphate-buffered saline for 2 hours, and co-stained successively using primary antibodies against GPC3 (Aviva, CA) and CD81 (GeneTex, TX). GPC3 rabbit antibody $(4 \mu \mathrm{g} / \mathrm{ml})$ was incubated overnight at $4^{\circ} \mathrm{C}$, washed, and followed by incubation using CD81 hamster antibody $(1.25 \mu \mathrm{g} / \mathrm{ml})$ for 2 hours under room temperature. Two fluorescent conjugated secondary antibodies were goat-antirabbit (Alexa 488) at a dilution of 1:500 and goat antihamster (Cy3) antibody at a dilution of 1:1000. Sections were counterstained with nuclear DNA probes DRAQ5 (Biostatus Limited, UK) at a dilution of 1:1000. Images were visualized using Nikon Eclipse confocal fluorescent microscope.

\section{Results}

\section{GPC3 Protein and RNA Level Increase during Liver Regeneration}

During rat liver regeneration following $\mathrm{PHx}$, different cell types proliferate at different times and the entire process 

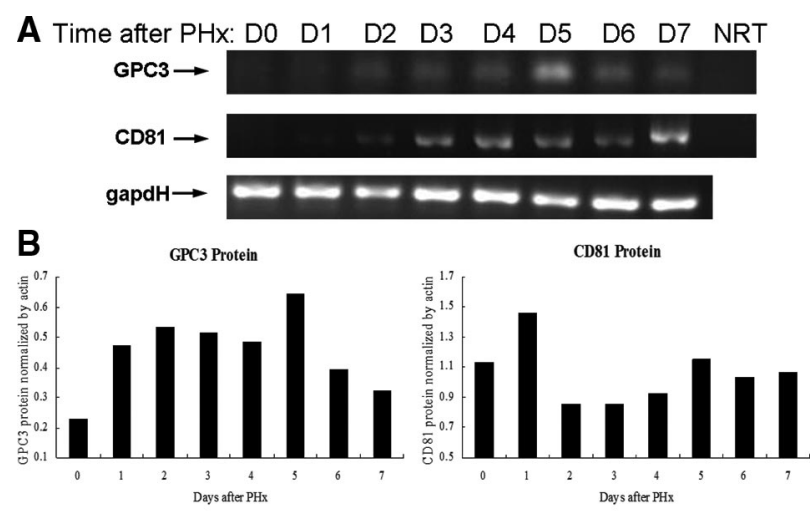

C

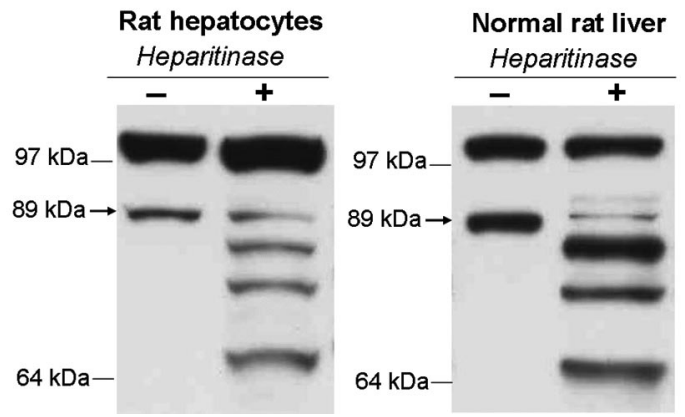

Figure 1. RNA and protein levels of GPC3 and CD81 increase during liver regeneration. A: Semiquantitative RT-PCR results show that GPC3 and CD81 expression are up-regulated in PHx after day 2. GAPDH: loading control. NRT: non-RT negative control. B: GPC3 and CD81 protein levels, normalized by actin, increase from day 2 after PHx with a peak expression at day 5 . C: Western blotting results of GPC3 protein extract from freshly isolated normal rat hepatocytes and normal rat liver tissue treated with heparitinase to eliminate HS chains. See details in Materials and Methods. The arrow indicates the 89-kd glycosylated form of GPC3.

usually finishes within 7 to 10 days. To study the role of GPC3 in liver regeneration, we first investigated the mRNA and protein levels of GPC3 during this process. Semiquantitative RT-PCR results showed that GPC3 expression increases from day 2 after rat $\mathrm{PHx}$ with a peak at day 5 (Figure $1 \mathrm{~A})$. Western blot analysis revealed that GPC3 protein started increasing from day 2 after $\mathrm{PHx}$, peaked at day 5, and decreased at day 6 (Figure 1B), corresponding with the RT-PCR results. It is reported that the core protein of GPC3 is $67.5 \mathrm{kd}$ in size. However, in our Western blotting an 89-kd band was detected by the rabbit anti-mouse GPC3 polyclonal antibody. In view of the fact that GPC3 is highly glycosylated, we treated the protein samples from freshly isolated rat hepatocytes as well as normal rat liver extract with heparitinase to eliminate HS chains. After a 3-hour treatment with heparitinase, the intensity of the $89-k d$ protein band diminished and a series of bands appeared, including the 67-kd GPC3 core protein band (Figure 1C). The multiple protein bands that appeared after heparitinase treatment may be due to incomplete digestion. These results are consistent with the reported high glycosylation levels of the 89-kd protein band.

\section{GPC3 Increases at the End of Hepatocyte Proliferation}

We further investigated GPC3 in hepatocyte cultures. Rat hepatocytes were isolated from normal rat liver and

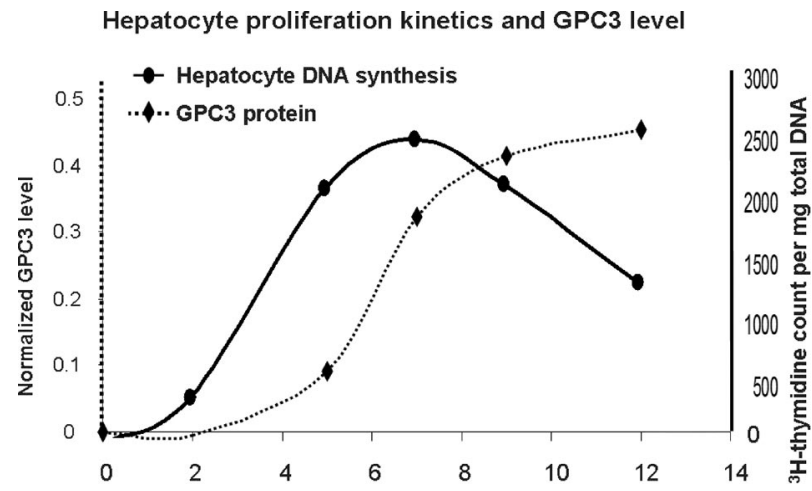

Figure 2. GPC3 levels increase when DNA synthesis stops in rat hepatocyte culture. $\left[{ }^{3} \mathrm{H}\right]$ thymidine incorporation per mg total DNA (solid line) and GPC3 level (dotted line) for different time points in hepatocyte culture are shown.

incubated with HGF and EGF to stimulate cell proliferation in culture. DNA synthesis was detected using $\left[{ }^{3} \mathrm{H}\right]$ thymidine incorporation assay, and hepatocyte proliferation $\left(\left[{ }^{3} \mathrm{H}\right]\right.$ thymidine count per mg DNA) was determined (Figure 2). The $\left[{ }^{3} \mathrm{H}\right]$ thymidine incorporation assay shows that there is a peak of hepatocyte proliferation at day 7 , and then DNA synthesis slows down. Western blotting showed that GPC3 levels, normalized by $\beta$-actin control, start increasing around day 7 (Figure 2), which correlated with the beginning of decrease in hepatocyte DNA synthesis.

\section{Knocking Down GPC3 by Morpholino Oligos Promotes Hepatocyte Growth in Culture}

To further determine the role of GPC3 in liver regeneration, Morpholino oligos were used to knock down GPC3 protein expression in hepatocyte cultures. Morpholino oligos against GPC3 were commercially synthesized and added together with Endo-Porter reagent at days 0 and 4 in hepatocyte cultures. To study the growth and proliferation of hepatocytes, $\left[{ }^{3} \mathrm{H}\right]$ thymidine counts and total DNA were collected for each time point. Growth curves were created according to $\left[{ }^{3} \mathrm{H}\right]$ thymidine counts (Figure $3 \mathrm{~A}$ ) or total amount of DNA (Figure 3B) for hepatocytes with GPC3 Morpholino oligos or only with Endo-Porter control. In cultures with Morpholinos, both $\left[{ }^{3} \mathrm{H}\right]$ thymidine counts and total DNA increase at day 6 , when cell proliferation in control cultures slows down. Western blotting results showed that GPC3 protein is reduced at days 4 and 6 in hepatocytes cultured with GPC3 Morpholino oligos but not with Endo-Porter control (Figure 3C).

\section{GPC3 and CD81}

To screen for proteins that might interact with GPC3, a yeast two-hybrid assay was performed. The details of clone isolation and characterization are given in Materials and Methods. Several interesting genes were found, such as tyrosine kinase substrate (Hrs), prostaglandin D2 synthase (Ptgds), $\alpha$-2-HS-glycoprotein (fetuin), vitamin D-binding protein, splicing factor (SRp20), ribosomal protein S2 (Rps2), ribosomal protein L29, T-cell differen- 
A

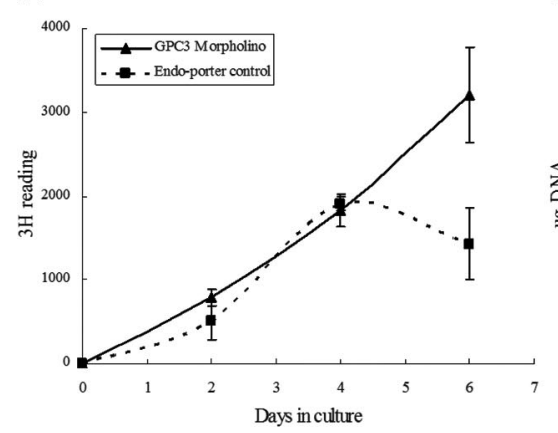

B

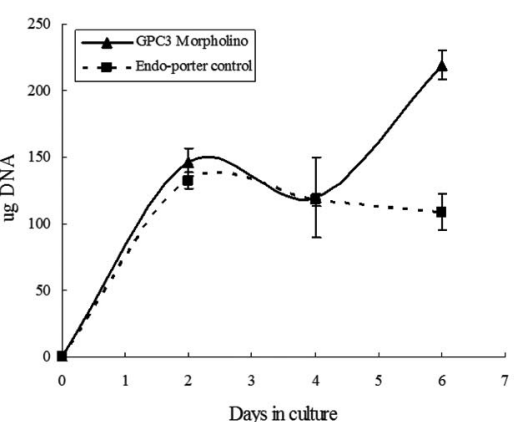

C

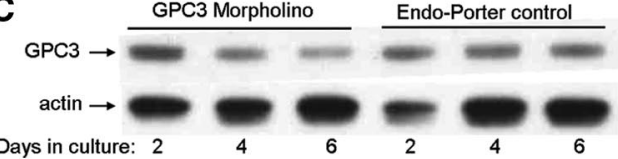

Figure 3. Effect of GPC3 Morpholino and Endo-Porter control on $\left[{ }^{3} \mathrm{H}\right]$ thymidine uptake $(\mathbf{A})$ and total DNA $(\mathbf{B})$ in rat hepatocyte cultures. All experiments were performed in triplicate; SE is indicated by the error bars. GPC3 Morpholino promoted hepatocyte growth at day 6. C: GPC3 protein is knocked down in cell cultures treated with GPC3 Morpholino oligos but not with Endo-Porter control, shown by Western blotting. tiation protein 2(Mal2), 5,10-methenyltetrahydrofolate synthetase (Mthfs), $\alpha$-1-inhibitor III, argininosuccinate synthetase (Ass), afamin (Afm), and CD81. Hrs is a gene producing a protein that is a substrate of hepatocyte growth factor-regulated tyrosine kinase (MET). ${ }^{30}$ The other proteins identified are either circulating in the plasma (fetuin, afamin), located in the cytosol (Hrs, vitamin D-binding protein), or having no apparent connection in location and function. Since GPC3 is a cell surface protein, we decided to focus on potentially interacting proteins that were also located on the plasma membrane. Thus we focused on CD81, also known as TAPA-1 (target of antiproliferative antibody 1), because it is a cell surface tetraspanin and reported to be involved in a variety of biological responses including inhibition of growth and proliferation. ${ }^{21}$ In the liver, previous research revealed that CD81 interacts with HCV glycoprotein E2, assisting with HCV entry into hepatocytes. ${ }^{23}$ The role of CD81 in liver growth regulation has not been previously investigated. As a first step, we assayed CD81 RNA and protein levels during liver regeneration. The results of semiquantitative RT-PCR and Western blotting showed that RNA and protein levels of CD81 start increasing from day 2 (Figure 1, A and B), which corresponds with changes of GPC3.

\section{GPC3 Co-Immunoprecipitates with CD81}

The yeast two-hybrid assay suggested that CD81 interacts with GPC3. To further test this finding, we performed a co-immunoprecipitation assay. Total protein was extracted from frozen normal rat liver, and CD81 was immunoprecipitated with anti-CD81 antibody or hamster IgG as binding control. The immunoprecipitated material was then probed for the presence of GPC3 using an anti-GPC3 polyclonal antibody. Figure 4 shows that the 89-kd band of GPC3 co-immunoprecipitates with CD81 but not the control IgG.

\section{Localization of GPC3 and CD81 in Hepatic Tissues by Immunofluorescence}

To further study the intracellular localization of GPC3 and CD81 in the liver, co-immunofluorescence was performed. Frozen sections of liver after PHx were doublelabeled with primary antibodies against GPC3 and CD81 and visualized using a confocal microscope to determine the localization of both proteins. Our results showed that both GPC3 and CD81 localize cell membranes during liver regeneration. At day 2 after $\mathrm{PHx}$, based on cell morphology, strong signal of colocalization appeared on hepatocyte plasma membrane while at day 6 , the colocalization signal appeared mainly along the sinusoids (Figure 5). It has been previously reported ${ }^{17}$ that during the rat liver regeneration, rat hepatocytes start proliferating first and finish proliferation around day 2, followed by the proliferation of other cell types. The whole process lasts about 5 to 7 days. Therefore, the colocalization of GPC3 and CD81 at day 2 may coincide with the termination of hepatocyte proliferation, while the colocalization on non-parenchymal cells at day 6 may reflect involvement with the cessation of proliferation of nonparenchymal cell types. Western blotting results (Figure 1B) also revealed that there was increase of GPC3 at day 2 and CD81 at day 1 after $\mathrm{PHx}$, followed by another peak around day 5 .

\section{Discussion}

In this study, we showed that GPC3 mRNA and protein increase in a time frame that coincides with the termination of proliferative activities of either hepatocytes (day 2 after $\mathrm{PHx}$ and days 8-12 in culture) or non-parenchymal cells (days 5-6 after PHx). These results and the previous

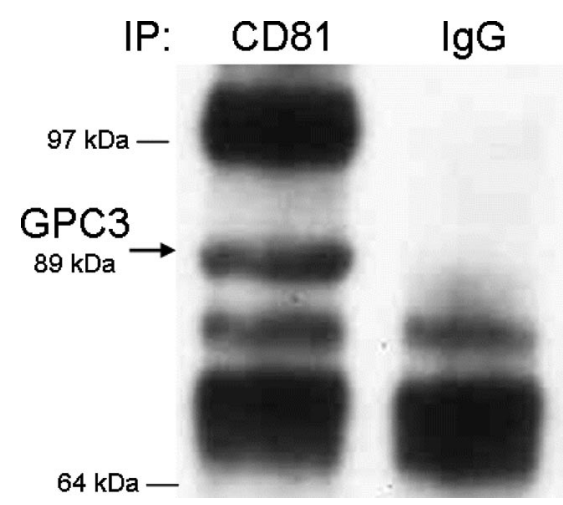

Figure 4. Co-immunoprecipitation of CD81 and GPC3. The rat liver lysates without heparitinase treatment were incubated with anti-CD81 monoclonal antibody or control IgG, followed by precipitation with agarose $A / G$ plus beads. Precipitates were separated by Western blotting and probed with anti-GPC3 rabbit polyclonal antibody. Arrow indicates 89-kd GPC3 protein. 


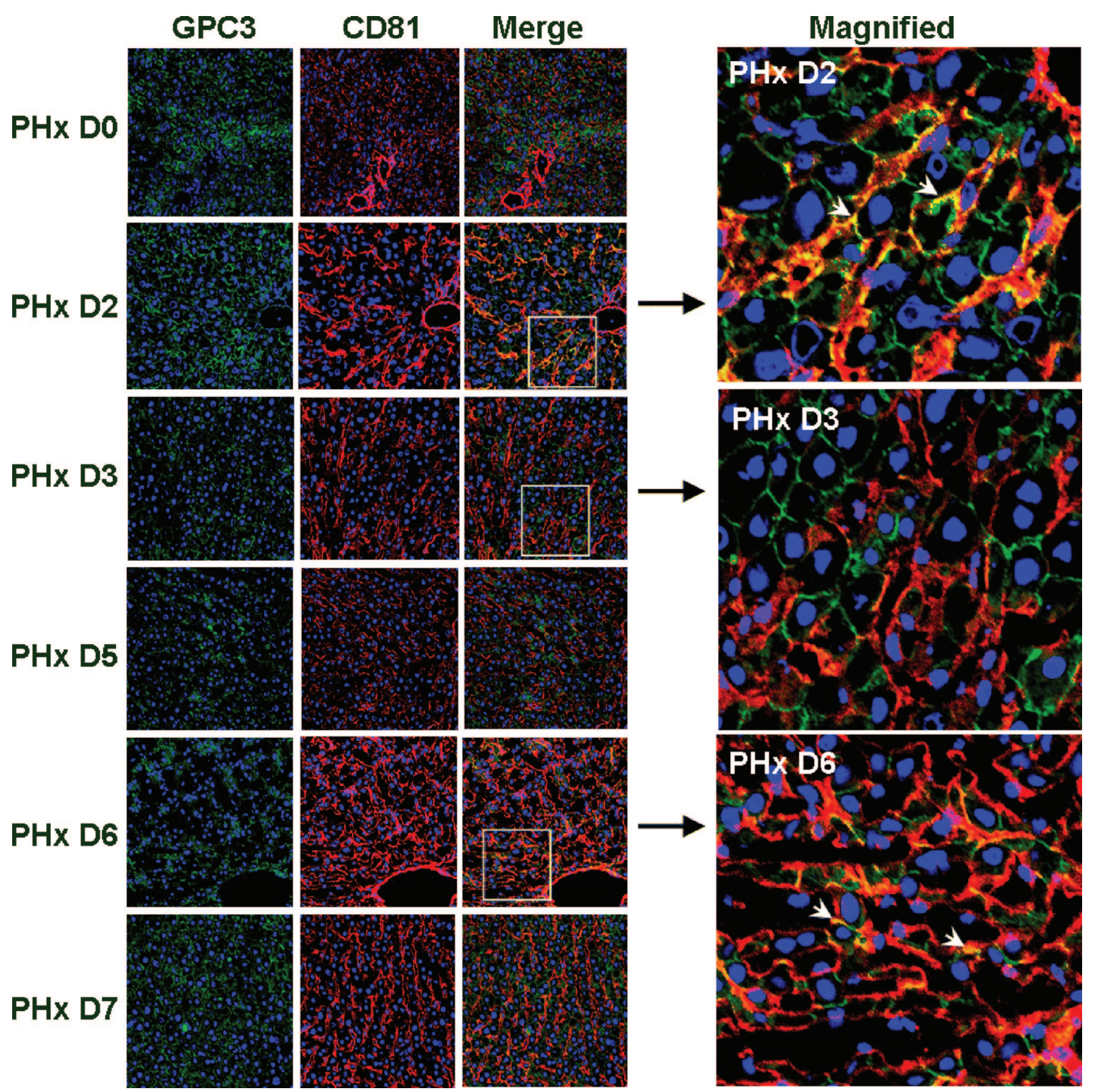

Figure 5. Immunofluorescence analysis of GPC3 and CD81 expression in rat liver regeneration process. Olympus Fluoview 500 confocal microscope was used to visualize the images; original magnification, $\times 200$. GPC3 (green), CD81 (red), and DRAQ5 nuclease staining (blue) are shown. Colocalization signals at days 2 and 6 after PHx are shown in the merged images (white arrowheads in magnified image). For details, see Results.

reports about GPC3 mutation in Simpson-Golabi-Behmel syndrome and organomegaly in GPC3 knockout mice are consistent with our hypothesis that GPC3 plays an overall growth inhibitory role, at least in liver regeneration and hepatocyte proliferation. The studies with patients suffering from SBGS syndrome also suggest that GPC3 is involved in regulation of growth of mesenchymal tissues, including muscle and bone. This correlates with the changes observed with GPC3 at day 5 and thereafter following $\mathrm{PHx}$, which involve proliferation of non-hepatocytic cells types, such as stellate cells and endothelial cells. ${ }^{17}$
The yeast two-hybrid assay suggests that GPC3 interacts with CD81, which is a cell membrane tetraspanin, a finding that we validated by co-immunoprecipitation. Further study showed that CD81 mRNA and protein increase in grossly the same time frame after PHx. Co-immunofluorescence results show that GPC3 and CD81 colocalize at days 2 and 6 after PHx, indicating an important regulator interaction between the two proteins. At day 2 after $\mathrm{PHx}$, strong signal of colocalization of GPC3 and CD81 appeared mainly on the cell membrane of hepatocytes. This may coincide with the termination of hepatocyte proliferation. At day 
6 after $\mathrm{PHx}$ when all of the other cells are finishing proliferation and arrangement, the colocalization signal appeared mainly along the sinusoids. These results suggest a potential regulatory role involving an interaction of CD81 and GPC3 at times in which separate cellular populations in the liver (hepatocytes at day 2 and non-parenchymal cells at days 5-7) cease to proliferate.

In view of our results and the studies with SimpsonGolabi-Behmel syndrome in humans and knockout mice, the high levels of GPC3 observed in human hepatocellular carcinoma ${ }^{9}$ are puzzling. We also observed, in rat liver tumors generated by DEN, that different levels of GPC3, some absent and some strikingly elevated, were seen (data not shown). ${ }^{9}$ The elevated expression of GPC3 seen in liver cancer may reflect the fact that the neoplastic hepatocytes attempt to raise their level of GPC3 as a built-in hepatocyte feedback aimed to stop their proliferation. Hepatocellular carcinoma cells, however, have lost their capacity to respond to this growth termination signal. Recent studies have also shown that GPC3 is elevated in hepatic progenitor cells (oval cells) in situations when liver regeneration is stimulated and hepatocyte proliferation is blocked. ${ }^{31}$ It is also possible that in those studies GPC3 may act as a growth regulator for hepatic progenitor cells.

In general, GPC3 has been reported to interfere with different pathways and growth factors and has a tissue- and stage-specific role in development and tumor growth. ${ }^{32}$ There are several glypicans and syndecans that could interact with growth regulatory pathways. It has been reported that heparan sulfate proteoglycans interact with fibroblast growth factors, bone morphogenetic proteins, Wnt pathway, and others. ${ }^{33,34}$ Those heparan sulfate proteoglycans could act as coreceptors, which regulate the binding affinity of these growth factors to their receptors. ${ }^{35}$ Therefore, it would be very interesting to investigate the role of GPC3 and other heparan sulfate proteoglycans in liver regeneration and hepatocyte growth and proliferation.

The interaction of GPC3 and CD81 is intriguing. One could speculate that the negative regulatory effects of GPC3 may be mediated via CD81. Since the latter is known to be a major portal of entry of HCV into hepatocytes, ${ }^{23}$ the finding raises the possibility that the HCV interaction with CD81 may disrupt a major growth regulatory pathway for hepatocytes. In view of the welldocumented but poorly understood induction of high frequency of hepatocellular carcinomas by HCV, this possibility is worthy of further exploration.

\section{Acknowledgments}

We thank Weike Lai for advice with the yeast two-hybrid assays and Dr. Donna Stolz for helpful suggestions on immunofluorescence.

\section{References}

1. Fransson LA: Glypicans. Int J Biochem Cell Biol 2003, 35:125-129

2. Song $\mathrm{HH}$, Filmus $\mathrm{J}$ : The role of glypicans in mammalian development. Biochim Biophys Acta 2002, 1573:241-246

3. De Cat B, David G: Developmental roles of the glypicans. Semin Cell Dev Biol 2001, 12:117-125

4. Veugelers M, De Cat B, Ceulemans H, Bruystens AM, Coomans C Durr J, Vermeesch J, Marynen P, David G: Glypican-6, a new member of the glypican family of cell surface heparan sulfate proteoglycans. J Biol Chem 1999, 274:26968-26977

5. Chen RL, Lander AD: Mechanisms underlying preferential assembly of heparan sulfate on glypican-1. J Biol Chem 2001, 276:7507-7517

6 . Filmus J: Glypicans in growth control and cancer. Glycobiology 2001 , 11:19R-23R

7. Iglesias BV, Centeno G, Pascuccelli H, Ward F, Peters MG, Filmus J, Puricelli L, de Kier Joffe EB: Expression pattern of glypican-3 (GPC3) during human embryonic and fetal development. Histol Histopathol 2008, 23:1333-1340

8. Nakatsura T, Kageshita T, Ito S, Wakamatsu K, Monji M, Ikuta Y, Senju $\mathrm{S}$, Ono T, Nishimura Y: Identification of glypican-3 as a novel tumor marker for melanoma. Clin Cancer Res 2004, 10:6612-6621

9. Luo JH, Ren B, Keryanov S, Tseng GC, Rao UN, Monga SP, Strom S Demetris AJ, Nalesnik M, Yu YP, Ranganathan S, Michalopoulos GK: Transcriptomic and genomic analysis of human hepatocellular carcinomas and hepatoblastomas. Hepatology 2006, 44:1012-1024

10. Yamauchi N, Watanabe A, Hishinuma M, Ohashi K, Midorikawa $Y$, Morishita Y, Niki T, Shibahara J, Mori M, Makuuchi M, Hippo Y, Kodama T, Iwanari H, Aburatani H, Fukayama M: The glypican 3 oncofetal protein is a promising diagnostic marker for hepatocellular carcinoma. Mod Pathol 2005, 18:1591-1598

11. Filmus J, Capurro M: Glypican-3 and alphafetoprotein as diagnostic tests for hepatocellular carcinoma. Mol Diagn 2004, 8:207-212

12. Farooq M, Hwang SY, Park MK, Kim JC, Kim MK, Sung YK: Blocking endogenous glypican-3 expression releases Hep 3B cells from G1 arrest. Mol Cell 2003, 15:356-360

13. Hughes-Benzie RM, Pilia G, Xuan JY, Hunter AG, Chen E, Golabi M, Hurst JA, Kobori J, Marymee K, Pagon RA, Punnett HH, Schelley S, Tolmie JL, Wohlferd MM, Grossman T, Schlessinger D, MacKenzie AE: Simpson-Golabi-Behmel syndrome: genotype/phenotype analysis of 18 affected males from 7 unrelated families. Am J Med Genet 1996, 66:227-234

14. Pellegrini M, Pilia G, Pantano S, Lucchini F, Uda M, Fumi M, Cao A, Schlessinger D, Forabosco A: Gpc3 expression correlates with the phenotype of the Simpson-Golabi-Behmel syndrome. Dev Dyn 1998, 213:431-439

15. Cano-Gauci DF, Song HH, Yang H, McKerlie C, Choo B, Shi W, Pullano R, Piscione TD, Grisaru S, Soon S, Sedlackova L, Tanswell AK, Mak TW, Yeger H, Lockwood GA, Rosenblum ND, Filmus J: Glypican-3-deficient mice exhibit developmental overgrowth and some of the abnormalities typical of Simpson-Golabi-Behmel syndrome. J Cell Biol 1999, 146:255-264

16. Michalopoulos GK, DeFrances MC: Liver regeneration. Science 1997, 276:60-66

17. Michalopoulos GK: Liver regeneration. J Cell Physiol 2007, 213: 286-300

18. Block GD, Locker J, Bowen WC, Petersen BE, Katyal S, Strom SC, Riley T, Howard TA, Michalopoulos GK: Population expansion, clonal growth, and specific differentiation patterns in primary cultures of hepatocytes induced by HGF/SF: EGF and TGF alpha in a chemically defined (HGM) medium. J Cell Biol 1996, 132:1133-1149

19. Higgins G, Anderson R: Experimental pathology of liver: restoration of liver of the white rat following partial surgical removal. Arch Pathol 1931, 12:186-202

20. Oren R, Takahashi S, Doss C, Levy R, Levy S: TAPA-1, the target of an antiproliferative antibody, defines a new family of transmembrane proteins. Mol Cell Biol 1990, 10:4007-4015

21. Levy S, Todd SC, Maecker HT: CD81 (TAPA-1): a molecule involved in signal transduction and cell adhesion in the immune system. Annu Rev Immunol 1998, 16:89-109

22. Yang X, Kovalenko OV, Tang W, Claas C, Stipp CS, Hemler ME: Palmitoylation supports assembly and function of integrin-tetraspanin complexes. J Cell Biol 2004, 167:1231-1240

23. Cocquerel L, Kuo CC, Dubuisson J, Levy S: CD81-dependent 
binding of hepatitis C virus E1E2 heterodimers. J Virol 2003, 77: 10677-10683

24. Stolz DB, Mars WM, Petersen BE, Kim TH, Michalopoulos GK: Growth factor signal transduction immediately after two-thirds partial hepatectomy in the rat. Cancer Res 1999, 59:3954-3960

25. Seglen PO: Preparation of isolated rat liver cells. Methods Cell Biol 1976, 13:29-83

26. Michalopoulos GK, Bowen WC, Zajac VF, Beer-Stolz D, Watkins S, Kostrubsky V, Strom SC: Morphogenetic events in mixed cultures of rat hepatocytes and nonparenchymal cells maintained in biological matrices in the presence of hepatocyte growth factor and epidermal growth factor. Hepatology 1999, 29:90-100

27. Tsuda H, Yamada S, Miyazono H, Morikawa K, Yoshida K, Goto F, Tamura JI, Neumann KW, Ogawa T, Sugahara K: Substrate specificity studies of Flavobacterium chondroitinase $\mathrm{C}$ and heparitinases towards the glycosaminoglycan-protein linkage region. Use of a sensitive analytical method developed by chromophore-labeling of linkage glycoserines using dimethylaminoazobenzenesulfonyl chloride. Eur J Biochem 1999, 262:127-133

28. Summerton J: Endo-Porter: a novel reagent for safe, effective delivery of substances into cells. Ann NY Acad Sci 2005, 1058:62-75
29. Van Criekinge W, Beyaert R: Yeast two-hybrid: state of the art. Biol Proced Online 1999, 2:1-38

30. Raiborg C, Stenmark H: Hrs and endocytic sorting of ubiquitinated membrane proteins. Cell Struct Funct 2002, 27:403-408

31. Grozdanov PN, Yovchev MI, Dabeva MD: The oncofetal protein glypican-3 is a novel marker of hepatic progenitor/oval cells. Lab Invest 2006, 86:1272-1284

32. Filmus $\mathrm{J}$, Capurro M: The role of glypican-3 in the regulation of body size and cancer. Cell Cycle 2008, 7:2787-2790

33. Midorikawa Y, Ishikawa S, Imanari H, Imamura T, Sakamoto H, Miyazono K, Kodama T, Makuuchi M, Aburatani H: Glypican-3, overexpressed in hepatocellular carcinoma, modulates FGF2 and BMP-7 signaling. Int $J$ Cancer 2003, 103:455-465

34. Capurro MI, Xiang YY, Lobe C, Filmus J: Glypican-3 promotes the growth of hepatocellular carcinoma by stimulating canonical Wnt signaling. Cancer Res 2005, 65:6245-6254

35. Kleeff J, Ishiwata T, Kumbasar A, Friess $H$, Buchler MW, Lander AD, Korc M: The cell-surface heparan sulfate proteoglycan glypican-1 regulates growth factor action in pancreatic carcinoma cells and is overexpressed in human pancreatic cancer. J Clin Invest 1998, 102:1662-1673 